\title{
Balkanologie
}

Balkanologie Revue d'études pluridisciplinaires

Vol. IV, $n^{\circ} 1 \mid 2000$

Volume IV Numéro 1

\section{Les angoisses fin-de-siècle des intellectuels roumains : démocratie, conservatisme et antisémitisme}

Denise Rosenthal

\section{CpenEdition}

Journals

Édition électronique

URL : http://journals.openedition.org/balkanologie/778

DOI : 10.4000/balkanologie. 778

ISSN : 1965-0582

Éditeur

Association française d'études sur les Balkans (Afebalk)

Édition imprimée

Date de publication : 1 septembre 2000

ISSN : 1279-7952

Référence électronique

Denise Rosenthal, « Les angoisses fin-de-siècle des intellectuels roumains : démocratie, conservatisme et antisémitisme », Balkanologie [En ligne], Vol. IV, $n^{\circ} 1$ | 2000, mis en ligne le 20 juillet 2011, consulté le 17 décembre 2020. URL : http://journals.openedition.org/balkanologie/778 ; DOI : https://doi.org/10.4000/balkanologie.778

Ce document a été généré automatiquement le 17 décembre 2020.

(c) Tous droits réservés 


\title{
Les angoisses fin-de-siècle des intellectuels roumains : démocratie, conservatisme et antisémitisme ${ }^{1}$
}

\author{
Denise Rosenthal
}

\section{Introduction: une sociologie des origines culturelles de la vie politique roumaine}

La fin du siècle étant déjà une réalité, le profil de l'intellectuel roumain démocrate vivant à l'époque critique de la transition mérite un réexamen. Depuis l'analyse significative de Katherine Verdery, le rôle de l'intellectuel roumain dans la période de la transition est devenu extrêmement compliqué 2 . Cela est vrai en raison du rôle de l'intellectuel en tant qu'opposant officiel sous le pouvoir de Ion Iliescu (1990-1996) d'une part, et sous celui d'Emil Constantinescu (1996-2000) d'autre part. Ces fonctions sont enracinées dans le rapport entre les intellectuels roumains et leur environnement: la société sud-est européenne. À l'exception notable des thèses en faveur du libéralisme, des valeurs bourgeoises, à savoir de la modernité (opposées aux thèses traditionalistes), de Stefan Zeletin et d'Eugen Lovinescu, on constate dans ce type de société l'absence d'une tradition d'élites politiques formées à une pensée rationaliste cartésienne bien définie ${ }^{3}$. En conséquence, elle doit reposer sur la culture pour palier ce manque. L'histoire des dirigeants politiques roumains a révélé un tel modèle en particulier depuis la génération des intellectuels libéraux-nationalistes du Paşoptişti de 1848. C'est à dire que la culture, en tant qu'embryon du nationalisme et de la construction nationale, et ses tendances traditionalistes, a supplanté le pragmatisme des arguments politiques occidentaux soutenant, en termes wéberiens ${ }^{4}$, la société bureaucratique et rationnelle occidentale, avec une large étendue de critères culturels, mobilisant l'histoire, la religion et occasionnellement le mysticisme comme paradigmes de légitimation (à savoir l'autochtonie et l'orthodoxie ${ }^{5}$ qui conduisirent ultérieurement au missionarisme mystique des légionnaires de l'entre-deux-guerres). 
2 Lorsque la culture devient la source des idées politiques et que les personnalités culturelles occupent les positions politiques majeures, la culture et la politique deviennent des instruments et des réalités interchangeables. Les poètes, les philosophes, les écrivains et les comédiens, dénués de traditions politiques et sociales enracinées dans la lignée des Lumières de la modernité démocratique et séculaire, deviennent des hommes politiques et détiennent les rênes de la destinée du pays. Comme nous le montrerons dans cette étude, dans ces circonstances, la politique ellemême fluctue à l'intérieur de valeurs culturelles s'inscrivant dans la religion et l'histoire nationale. Ce processus s'est déroulé dans la phase extraordinaire de la transition après 1989 où on s'est efforcé d'affronter la modernité occidentale retranchée dans le néo-libéralisme et la globalisation croissante.

3 Par conséquent, il est devenu important d'analyser, dans le contexte roumain, le discours de l'intellectuel humaniste-cum-homme politique.

4 La première raison pour examiner le monde des intellectuels roumains est l'idée centrale de cette étude qu'il y a une équation est-européenne illusoire entre les valeurs de l'anticommunisme et celles de la démocratie. À travers une analyse de positionnements discursifs variés, nous révélerons certaines des valeurs les plus frappantes de l'élite politique culturelle soutenant le pouvoir au cours de la période 1996-2000. Bien que largement louée lorsqu'elle arriva au pouvoir, cette élite comprend principalement des personnalités dont le savoir est basé sur un ethos anticommuniste et une formation intellectuelle philosophique et littéraire humaniste (moins en sciences sociales). Toutefois, ce fondement ne correspond pas nécessairement à un engagement politique démocratique et libéral soutenu dans son acception occidentale. $\mathrm{Au}$ contraire, nous démontrerons que ce conglomérat d'anticommunisme et de libéralisme est fondamentalement conservateur, tandis que la démocratie est réduite à l'état de "gauchisme" repoussé dans les oubliettes de l'histoire.

5 Parmi les personnalités et les hommes politiques qui souscrivent à cette formule de la discipline intellectuelle figurent Andrei Pleşu, critique d'art qui fut ministre-adjoint de la culture dans le gouvernement provisoire de 1990 ; Mihai şora, philosophe, ministreadjoint de l'Éducation en 1990, devenu ministre-adjoint des Affaires Étrangères entre 1996 et 1999 ; Ion Caramitru, ministre-adjoint de la Culture, comédien renommé, ainsi que Nicolae Manolescu et Ana Blandiana, le premier critique littéraire célèbre et homme politique libéral jusqu'en juillet 2000, dirigeant du Partidul National Liberal (PNL), la seconde écrivain et poète réputée dirigeante du Partidul Alianţei Civice (PAC). À leurs côtés, il faut mentionner le Grupul pentru Dialog Social (GDS), rassemblant des intellectuels humanistes de centre-droit prétendant publiquement représenter la société civile roumaine, ce qui reste à prouver. Le GDS semble fonctionner comme l'un des groupes d'intérêts et des centres épistémologiques les plus significatifs, travaillant aux objectifs des intellectuels liés aux valeurs anticommunistes, nationales, libérales, conservatrices et même monarchistes, et remplissant occasionnellement les fonctions de lobby, ainsi que de conseil consultatif présidentiel officieux. De la sorte, la Roumanie a réintégré sa tradition précommuniste de la vie politique au substrat culturel. Le continuum entre culture et politique est rétabli à travers des arguments légitimés par l'histoire et la religion, et non les principes rationnels du mode de gouvernement et de la politique des Lumières ${ }^{6}$.

6 La seconde raison pour laquelle ce phénomène mérite d'être analysé résulte de l'observation que bien que les hommes politiques issus du monde culturel se qualifient 
eux-mêmes de libéraux, démocrates et pro-européens, leurs propositions politiques soutiennent principalement le conservatisme roumain, élitiste, religieux et parfois pseudo-démocratique. Le conservatisme roumain classique qui tire son essence du conservatisme européen, met en avant les valeurs roumaines spécifiques, et en conséquence, réagit contre le progrès, le libéralisme et la modernité, avec la tendance que l'illustration ethnique se transforme en "ethnocratie"7. Le conservatisme roumain privilégie l'"élite" contre la "masse", insiste sur les valeurs ethniques et culturelles roumaines et défend la monarchie ${ }^{8}$. Tandis que certains intellectuels conservateurs expriment leur allégeance au libéralisme, la notion de républicanisme, comme celle de démocratie, est un concept compromis, un symbole utilisé par la gauche nostalgique du communisme (Partidul DemocraŢiei Sociale din România, PDSR), le centre-gauche technocratique (Partidul Democrat, PD) et les ultranationalistes (Partidul România Mare, PRM ; Partidul UnităŢii NaŢionale Române, PUNR et "Vatra Românească»). De surcroît, il faut mentionner que la république, en tant que forme d'État, n'a pas de tradition en Roumanie hormis sous le régime communiste. Néanmoins, le fait que la Roumanie soit une république aujourd'hui est considéré comme un héritage du régime communiste dans la mesure où la constitution républicaine de 1991 a été élaborée sous l'hégémonie du Front de Salut National (FSN), devenu plus tard le PDSR. Le PDSR, le PRM et le PUNR ont formé ultérieurement la coalition regroupant les ex-communistes et (ultra)nationalistes, connue également sous el nom de coalition "rouge-brun". Excepté cela, la république ne dispose pas d'une nouvelle légitimité politique positive. Sous les différents gouvernements entre 1990 et 1996, l'opposition, les intellectuels mentionnés ici (des groupes libéraux divers, Partidul NaŢional-Ţărănesc Creştin-Democrat - PNŢCD - et certaines voix du GDS), se sont prononcés sans ambiguïté en faveur de la monarchie, ce qui n'est plus aussi prégnant aujourd'hui ${ }^{9}$.

7 Néanmoins, tous les courants de pensée, monarchiste ou libéral, font valoir un concept de la démocratie confus, principalement parce que les démocrates-libéraux roumains se définissent eux-mêmes en opposition aux ultranationalistes, et non sur la base de l'axiome de la démocratie libérale lui-même. De fait, la démocratie libérale englobe des signifiants politiques occidentaux spécifiques de la période des Lumières qui n'ont jamais pénétrer le temps et l'espace des sociétés est-européennes d'une manière synchronique ou structurelle. Aujourd'hui, selon la réévaluation du concept de Mitteleuropa de Timothy Garton Ash ${ }^{10}$, la grande majorité des intellectuels de l'Europe du centre-est revendiquent l'appartenance à l'Europe de la Hongrie, de la Pologne et de l'ancienne Tchécoslovaquie sur les plans de la culture et de la civilisation: la Renaissance, la Réforme, les Lumières, la Révolution française et la Révolution industrielle. En conséquence, ces intellectuels considèrent que l'Europe du centre-est se distingue foncièrement des régions situées plus à l'est (la Russie et les Balkans ottomans, ensemble auquel les principautés roumaines appartenaient pour l'essentiel jusqu'en 1877), dont les frontières occidentales marquent les frontières orientales de l'Europe ${ }^{11}$. L'écrivain tchéco-français, Milan Kundera, s'interrogeait carrément : «que signifie l'Europe pour un Hongrois, un Tchèque, un Polonais? ", considérant les pays de Višegrad comme une partie de l'esprit occidental et de la chrétienté catholique romaine en opposition à l'Europe du sud-est et la Russie. «Pour eux [la Hongrie, la République tchèque et la Pologne], le mot Europe ne représente pas un phénomène géographique, mais une notion spirituelle synonyme du mot "Occident". ${ }^{12}$ Dans ce contexte, la démocratie devient alors une notion variable entre l'est et l'ouest, dépendant de la rupture ou de l'interpénétration entre les pratiques politiques et les 
cultures des parties orientale et occidentale de l'Europe. Ainsi, les arguments soutenant l'idée que la Roumanie postcommuniste s'approche de la démocratie occidentale sous le seul prétexte du génie anticommuniste roumain, comme de nombreux intellectuels démocrates-libéraux autoproclamés l'affirment aujourd'hui, en se situant en opposition aux ultranationalistes, sont fallacieux. Cela est évident dès que l'on met à nu la position historique ambivalente de la Roumanie vis-à-vis du paradigme politique occidental ${ }^{13}$.

Les conditions cumulatives de la démocratie libérale comprennent le suffrage universel égalitaire, le pluralisme politique, les droits de l'homme, le respect de la diversité ethnique, culturelle et sociale, l'esprit de transparence institutionnelle et de responsabilité au sein d'une bureaucratie rationnelle, ainsi que des débats ouverts et critiques à l'intérieur d'une société civile active et réactive. En l'absence de ces facteurs, le système démocratique roumain manque de substance.

À la lumière de la source de légitimation, la culture, nous conclurons que les sentiments pro-européens des hommes politiques issus des milieux culturels sont plus orientés vers les valeurs culturelles que les valeurs politiques européennes, lesquelles, une fois reliées, sont confuses. Dans la mesure où il s'agit d'une étude critique des valeurs et des arguments discursifs et non d'une description fonctionnelle de la position des intellectuels roumains dans la sphère politique actuelle, il faut souligner que leurs messages sont hétérogènes et ambigus, c'est à dire qu'il n'y a pas de camps clairement dessinés, bien que certaines tendances soient réellement observables. Cependant, pour les besoins analytiques de notre propos, il faut préciser que les intellectuels roumains ont évolué sous l'influence de stimuli politiques intérieurs et extérieurs dans deux groupes principaux : les intellectuels culturels et les intellectuels politiques. Parfois, ces deux groupes s'opposent, parfois ils se rejoignent. En outre, il faut noter qu'aucun de ces groupes n'adhère strictement à un ensemble de principes politiques, ce qui accentue l'ambiguïté. De surcroît, des textes publiés par certains intellectuels montrent un conservatisme politique fondé sur la culture à l'œuvre et une perception roumaine particulière de la démocratie en concurrence avec le nationalisme ${ }^{14}$. La dernière partie abordera la polémique récente de l'“Holocauste contre Goulag", un test de démocratie face auquel de nombreux intellectuels démocrates et libéraux semblent avoir échoué. Malgré l'esprit démocratique de l'élite politique et culturelle de 1996, ses membres ont proposé peu d'alternatives libérales et démocrates aux fantômes du communisme ainsi qu'à l'ultranationalisme actuel en Roumanie ${ }^{15}$.

\section{Les intellectuels culturels : Les Rois-Philosophes et le conservatisme}

10 Examinant leurs positions après 1989 , on peut dire que les intellectuels démocrates souffrent d'une compréhension problématique du concept de démocratie, situation qui révèle en retour une tension entre orientations conservatrices, libérales et démocratiques. À partir de la moitié des années 1990, on discerne un nouveau groupe d'intellectuels. Il s'agit des intellectuels d'orientation libérale et démocrate engagés en politique, bien qu'ils soient situés dans un ensemble plus large d'intellectuels culturels agissant dans un univers roumain conservateur. Ici, la notion de génération intellectuelle renvoie à des valeurs fondamentales constituant la base d'un mouvement et non à l'âge des acteurs en question. Une génération peut rassembler des intellectuels d'âges divers qui sont unis par une axiologie, une ontologie, voire une méthodologie 
communes. Ainsi, l'analyse de leurs positions publiées révèle que les intellectuels culturels fournissent des arguments politiques par le biais de l'histoire et de la culture politisées (ce qui est courant en Roumanie), tandis que les intellectuels politiques sont engagés dans des analyses favorables à la démocratie basées sur les principes fondamentaux de la science politique libérale et démocrate (à savoir ceux des droits de l'homme et du règne du droit). Le profile psychologique et le type de formation des intellectuels roumains sont cruciaux pour comprendre le schisme politique actuel ainsi que la nouvelle guerre d'étiquettes affectant le milieu des intellectuels. D'une part, on trouve les intellectuels formés dans les traditions culturelles française et allemande classiques, reposant sur un fond de traditionalisme roumain et des engagements éthiques puissants, dont l'anticommunisme. Il s'agit principalement de l'ancienne garde des anticommunistes, en particulier de la période de la soviétisation, caractérisée par le radicalisme non communiste de ceux qui furent persécutés ou des prisonniers politiques. On les retrouve surtout parmi les membres des partis historiques, tel que le PNŢCD, ou encore le PNL ainsi que parmi les partis ou groupes libéraux apparus après 1989. Toutefois, Adrian Marino, critique littéraire pro-européen sur le plan politique, explique que ce groupe présente un autisme subtil face aux changements dans la société roumaine contemporaine: «les valeurs fondamentales dans lesquelles les prisonniers politiques croyaient et continuent de croire sont purement morales: intransigeance, caractère, sobriété, pureté, sévérité anticommuniste radicale $»^{16}$.

11 D'autre part, on trouve une génération plus récente d'intellectuels appartenant aux mêmes écoles de pensée allemande et française se distinguant par un engagement autodéclaré en faveur de la société civile, du libéralisme et de l'Europe. C'est la première différenciation parmi les intellectuels démocrates. Ils sont rassemblés essentiellement au sein du GDS : Gabriel Liiceanu, philosophe et directeur de la maison d'édition Humanitas, Andrei Pleşu, Nicolae Manolescu, Alexandru Paleologu, juriste et publiciste, Octavian Paler, journaliste et Horia-Roman Patapievici, physicien et écrivain. Les valeurs de cette seconde génération émanant des milieux culturels sont formées à partir du calque des années 1970 et 1980, bien que nombre d'entre eux affirment leur loyauté aux paradigmes conservateurs de l'entre-deux-guerres, tels que le monarchisme ou la "belle époque" roumaine. Après 1989, les membres de ce groupe ont inauguré un discours sur la politique mêlant arguments culturels et politiques. Certains d'entre eux sont rassemblés autour du "groupe Păltiniş", connu sous le nom de "disciples de Noica" ${ }^{17}$, en raison du culte voué à leur maître, l'illustre philosophe Constantin Noica. Ils forment un noyau épistémologique comprenant des personnalités clés comme Gabriel Liiceanu et Andrei Pleşu.

12 Le "groupe Păltiniş" représente une carte importante, quoiqu'épineuse, en raison du passé de Noica ainsi que de l'hégémonie politique de cette seconde génération culturelle au cours de la période 1996-2000. C'est pourquoi il est important de présenter leurs origines et leurs valeurs. En premier lieu, la biographie de Noica est marquée par son passé spirituel légionnaire ${ }^{18}$, sa coopération ambivalente avec le régime communiste, ainsi que par sa fascination pour un courant de pensée débutant avec l'ancien grec et la métaphysique et culminant dans la philosophie idéaliste allemande et la quête romantique-existentielle de "nouvelles valeurs" et d'un "homme nouveau" dans le cadre d'une démocratie ethnique. Il a traduit et publié avec dévouement Platon, Kant, Plotinus, Kierkegaard, Nietzsche, Freud et Heidegger. L'alternative métaphysique de Noica à la rationalité moderne (capitalisme) et au marxisme est dénommée 
" résistance à travers la culture » (et abstention de toute action politique) au sein d'une éducation particulière, "Paideia", et de valeurs nationales et traditionnelles s'inscrivant dans un missionarisme religieux chrétien orthodoxe, en tant que salut spirituel. Cette alternative valut à son travail d'être approché non seulement par des antiprotochronistes (le plus éminent étant Nicolae Manolescu) mais aussi par des protochronistes / (ultra)nationalistes, tels que Eugen Barbu, Ion Coja, Paul Anghel, Dan Zamfirescu. Bien qu'un esprit occidental puisse facilement comprendre que ces valeurs sont contradictoires avec une exigence démocratique, tel n'est pas le cas en Roumanie. De nos jours, les disciples de Noica et leur cercle diffusent le culte de sa personnalité, principalement dans des éditions non critiques de ses travaux, les plus souvent publiés par la maison d'édition Humanitas, de loin la plus prestigieuse de ce genre en Roumanie. Les partisans de Noica tentent d'établir de la sorte une généalogie des créateurs et philosophes non communistes roumains de valeur, ne tenant aucun compte de la felix culpa de l'entre-deux-guerres de ces intellectuels de renom ${ }^{19}$.

De surcroît, l'ensemble GDS / Humanitas / disciples de Noica constitue également le générateur épistémologique dominant des valeurs politiques et culturelles sous le gouvernement actuel. Le problème est que la plupart des intellectuels issus des milieux culturels et engagés en politique appartenant à cet ensemble (la seconde génération) sont les porteurs de valeurs conservatrices : élitistes, religieuses, et parfois pseudodémocratiques. Néanmoins, la plupart d'entre eux déclareront de tout leur cœur être attachés à la démocratie, au libéralisme et à l'Europe. C'est pourquoi le chercheur occidental, habitué lors de la période communiste à écouter avec optimisme les discours des dissidents et intellectuels éminents, peut croire sur parole le discours des intellectuels d'après 1989, mais lorsqu'il analysera leurs travaux, publiés principalement dans les quotidiens et les revues littéraires polémistes, et non dans des publications académiques ou consacrées à la recherche, il sera abasourdi par leurs déclamations ethno-religieuses et conservatrices explicites ou hésitantes.

Un intellectuel proéminent mais controversé, Horia-Roman Patapievici, nous offre un exemple éloquent de ce genre de perspective contradictoire ${ }^{20}$. Tandis qu'il se présente comme un démocrate-libéral pro-européen éclairé et que l'opinion roumaine le considère comme tel, Patapievici soutient d'un style Cioranien dans ses écrits journalistiques la suppression du suffrage universel égalitaire :

Dans l'histoire, les Roumains ne se prononcent pas directement, et lorsqu'ils se mettent à en parler (du suffrage universel égalitaire), ils ne profèrent que des stupidités. Je crois fermement que la garantie du progrès en Roumanie passe par un suffrage restreint: la Roumanie évoluera uniquement si le peuple, misera plebs, ne participe pas directement au processus de décision. ${ }^{21}$

En outre, Patapievici considère le multiculturalisme comme le "communisme américain", "le nazisme" et le "léninisme", affirmant que l'université américaine est une institution étroite d'esprit avec sa posture multiculturelle intégratrice :

Dans certains cercles aux États-Unis, on parlera de vos hauts faits culturels que si vous avez la chance d'être homosexuel, une femme, un noir ou un mexicain. En règle générale, il est important d'appartenir à une minorité possédant une identité agressive et qui s'est faite entendre à travers une campagne contre la dictature de la culture chrétienne blanche (...). En ce qui me concerne, ma carte de visite est la suivante : je suis blanc, roumain, chrétien orthodoxe et hétérosexuel; je suis fier d'appartenir à une culture à une civilisation fondée sur les valeurs chrétiennes, comme le sont les valeurs européennes (...). En termes de "politiquement correct", j'ai l'identité typique de la culture détestable du chrétien blanc et situé à droite. ${ }^{22}$ 
Patapievici considère le féminisme comme le "fascisme", et y adjoint des notions telles que «fil de fer barbelé ", « commissaires femmes » et «camps de concentration pour hommes ". Il rejette sur un ton moqueur les questions environnementales ${ }^{23}$, tandis que dans d'autres articles il suggère la suppression des syndicats ${ }^{24}$, présente la minorité hongroise comme impérialement arrogante et opposée à la « majorité appartenant à la bonne foi » ethnique roumaine ${ }^{25}$, jouant ainsi la carte dangereuse de la psychologie ethnique ${ }^{26}$.

Ce type de raisonnement a suscité des réactions variées, de la mise en garde de Nicolae Manolescu affirmant que Patapievici sympathisait avec des formulations antidémocratiques $^{27}$, aux ultranationalistes ou aux communistes orthodoxes (la distinction entre ces deux catégories demeure floue jusqu'à ce jour) qui ont rendu l'affaire plus problématique ${ }^{28}$. Cette dernière est devenue plus épineuse dans la mesure où il ne s'agit pas d'un débat noir et blanc entre communistes et anticommunistes. Élément important, Patapievici a été attaqué par les ultranationalistes/communistes pour avoir représenté de façon injurieuse le peuple roumain. Cependant, il ne fut critiqué ni pour son libéralisme autoproclamé ni pour ses conceptions essentialistes du peuple roumain (qui sont autant dommageables que certaines des revendications essentialistes de ses détracteurs), son orthodoxisme ou encore son souhait de supprimer le suffrage universel égalitaire. En d'autres termes, une perspective essentialiste (les points de vue ultranationalistes et crypto-communistes) en a pourfendu une autre (les points de vue démocratiques douteux, conservateurs, élitistes et désobligeants). Toutefois, ces attaques ne peuvent pas être perçues uniquement comme des "invectives de l'extrême droite ou de l'extrême gauche $»^{29}$, ou comme une position « antimajoritaire » ou " antipopuliste » plutôt qu' « antidémocratique » ${ }^{30}$, dans la mesure où du point de vue démocrate et libéral, le suffrage universel égalitaire est une valeur générale de la démocratie moderne et non de quelque groupe extrémiste, ou sinon un « résidu communiste».

En présence de tels points de vue, le fait que Patapievici se déclare libéral et démocrate, et non conservateur ayant un rapport trouble aux valeurs démocratiques modernes, devient problématique. L'élision entre un ethos anticommuniste et un positionnement démocratique et libéral renforce la confusion profonde entre ces différentes valeurs existant dans l'imaginaire intellectuel roumain, permettant au conservatisme de garder la main haute et de s'opposer occasionnellement aux propositions démocratiques.

Revenons aux valeurs de l'ensemble GDS / Humanitas / disciples de Noica et soulignons leurs caractéristiques. Fondant leurs arguments et leurs opinions politiques dans la culture, ils ont rediffusé sans esprit critique les philosophes allemands renommés, tels que Friederich Nietzsche et Martin Heidegger, ainsi que la Jeune génération intellectuelle de l'entre-deux-guerres où figurent le mentor Nae Ionescu et des personnalités brillantes telles que Emil Cioran, Mircea Eliade et Constantin Noica. Ces écrits sont édités essentiellement par Humanitas ${ }^{31}$ et d'autres maisons d'édition, ces dernières penchant vers le nationalisme mystique (par exemple, la maison d'édition Anastasia, ayant publié Nae Ionescu) ${ }^{32}$. Ne pas informer le jeune public à propos d'Heidegger et de ses croyances politiques nazis, par exemple, peut conduire à des malentendus sur le travail de ce philosophe important et entraîner les nouvelles générations d'intellectuels sur le sentier de l'acceptation déformée et sans esprit critique de l'histoire des idées et des événements. De même pour Eliade, Cioran et Noica, au lieu de procéder à une approche critique et d'expliquer leurs attaches 
spirituelles et éthico-mystiques diverses au mouvement légionnaire, plutôt que leurs positions politiques ou criminelles, questions qui n'ont pas été soulevées sous le régime communiste, les éditeurs actuels préfèrent recourir à une forme d'amnésie historique, effaçant du même coup leur passé inconfortable, chose que Cioran n'accepta pas regrettant publiquement à Paris la "transfiguration" de sa jeunesse ${ }^{33}$. Après tout, la distinction entre les mondes "profane" et "mystique" n'est pas un trait du mouvement légionnaire roumain ou du nazisme, mais appartient intégralement à l'antipositivisme européen des années 1930, un courant de pensée qui anima non seulement Eliade en Roumanie, mais Heidegger en Allemagne, et tant d'autres intellectuels conservateurs non-fascistes de cette époque. Cet antipositivisme a au mieux constitué uniquement l'arrière-plan culturel ayant favorisé le développement ultérieur des solutions politiques antirationnelles et mystiques, sans former pour autant une théorie nazie ou du mouvement légionnaire, viciant ainsi prétendument le corpus entier de la philosophie d'Heidegger ou la mythologie d'Eliade ${ }^{34}$.

20 En fait, les intellectuels culturels souhaitent la résurrection de l'identité nationale perdue, écrasée par le régime communiste, et la revitalisation d'une tradition perdue. Leur préoccupation est d'éduquer la jeunesse dans les valeurs de la culture classique et de l'histoire roumaines fondée sur l'identité religieuse chrétienne orthodoxe et sociale, considérée comme la base de l'État roumain. Dans leur effort de délimiter leur nationalisme "modéré ", les intellectuels culturels le qualifient de "légitime » en opposition au « nationalisme exclusif, hégémonique » des ultranationalistes ${ }^{35}$.

\section{Les intellectuels politiques : les progressistes}

21 Une troisième génération d'intellectuels a également émergé. Au milieu des années 1990, les débuts de la désagrégation intellectuelle (située essentiellement au sein du GDS) ont commencé à désintégrer la seconde génération, non sur le plan actif et idéologique, mais professionnel et épistémologique. De la même façon que le monde universitaire occidental et nord-américain en particulier, était prêt et impatient d'accepter des esprits critiques de toute l'Europe de l'est par le biais de postes d'agrégés, de bourses universitaires, de professeurs invités, des programmes d'allocations, d'autres organismes éducatifs proposant d'autres alternatives, l'université roumaine, qui mériterait de faire l'objet d'une étude séparée, a commencé à introduire des penseurs politiques occidentaux de différents domaines, tels que la théorie politique, les relations internationales et la sociologie. On s'attendrait à trouver les nouvelles générations de jeunes intellectuels locaux orientés vers les idées occidentales de la démocratie libérale et les méthodes modernes de l'analyse en sciences sociales. Toutefois, les bonnes intentions de l'université roumaine sont limitées par des contraintes pratiques organisationnelles et le conservatisme de nombreux professeurs qui détiennent encore les mêmes chaires depuis 20 ou 30 ans. Cette troisième génération la plus récente est composée d'analystes politiques d'origines diverses. Parmi les plus significatifs figurent George Voicu, politologue et doyen de la faculté de sciences politiques à l'université de Bucarest ; Gabriel Andreescu, militant des droits de l'homme; Mihaela Miroiu, philosophe féministe et doyen de l'École nationale de sciences politiques et d'études administratives à Bucarest; Dan Pavel, écrivain et directeur du programme américain en Roumanie Project Ethnic Relations ; Andrei Cornea, intellectuel démocrate et linguiste spécialiste du grec ancien ; Alina Mungiu-Pippidi, analyste politique et dramaturge ; Stelian Tănase, politologue et 
directeur de la revue d'analyse politique Sfera Politicii ; et Renate Weber, militante des droits de l'homme, co-présidente avec Gabriel Andreescu du Comité Helsinki pour les droits de l'Homme en Roumanie. Certains intellectuels appartenant à cette troisième génération sont également membres du GDS et contribuent aux pages des revues suivantes : 22 (hebdomadaire du GDS), Sfera Politicii et Studii internationale ${ }^{36}$. Ceux qui ont bénéficié de telles expériences sont éduqués dans la tradition libérale-démocratique du pragmatisme anglo-américain, de l'empirisme et de la spécialisation. Néanmoins, ce groupe comme les autres ne représente pas un ensemble de principes politiques parfaits, mais un mélange d'idées.

Cette troisième génération peut être identifiée comme un groupe intellectuel politique émergeant (en roumain, politologi), ou de politistes, comme ils seront dénommés dans la tradition anglo-américaine. Néanmoins, il ne faudrait pas croire qu'un climat favorable à la science politique authentique a été créé de nos jours en Roumanie. En raison des modèles prémodernes, traditionnels de la société, renforcés par la logique corrompue du national-communisme, la société roumaine continue de fonctionner sous la catalyse de groupes cloisonnés, ou de prestige au sens de Weber. De ce fait, la concurrence ouverte, l'information libre, les revues mentionnées, le droit d'auteur, la recherche approfondie et l'édition académique, n'ont jamais fait l'objet d'une pratique systématisée. Plus grave encore, les commentaires et les essais sur des sujets politiques publiés dans la presse quotidienne ou les revues polémistes (un réel « journalisme avec des notes de bas de pages ${ }^{37}$ ) sont confondus avec la science politique en tant que telle. À la lumière de ces considérations, il est préférable de dénommer cette dernière génération d'intellectuels politiques, plutôt que de politologues. Différant des valeurs diffusées par les intellectuels culturels, les valeurs de ce dernier groupe sont associées à la promotion des droits de l'homme, la création et la fondation d'institutions propres à l'État de droit, une approche démocratique et universelle de toutes les minorités (dont les statuts peuvent être basés sur la nationalité, le genre et la sexualité), la signature de traités internationaux et l'intégration européenne. De surcroit, si les intellectuels culturels sont intéressés essentiellement par la reconstruction de l'identité nationale, les intellectuels politiques mettent l'accent sur la démocratie comme le tout premier des objectifs. Confrontés aux premiers, les intellectuels politiques considèrent que les intellectuels culturels vivent dans un univers nostalgique néoromantique, idéalisant l'histoire, l'idée d'Église nationale, le village originel idyllique et la Roumanie de l'entre-deux-guerres. Il est intéressant de noter que ce débat intellectuel a été analysé comme un cas appliqué du « débat entre libéraux et communautaristes dans la théorie politique anglo-américaine $»^{38}$, où la seconde génération des intellectuels culturels représenteraient les communautaristes et la troisième les libéraux.

Les valeurs des intellectuels politiques et culturels sont très importantes étant donné l'interface de leurs rôles politique et culturel épistémologiques qu'ils tiennent sous le pouvoir actuel. En outre, ces deux générations ont modelé l'opinion politique roumaine qui, sceptique à propos de la politique parlementaire, soutenait selon des enquêtes datant de 1999 l'Église à $88 \%$, l'armée à $75 \%$ et les partis politiques à $25 \%{ }^{39}$.

Ce qui demeure significatif dans le schisme intellectuel démocratique est la conception de la nation en termes ethniques par les intellectuels culturels (citoyenneté exclusive), tandis que les intellectuels politiques mettent l'accent sur sa définition civique (citoyenneté inclusive). Cela rend la question de la démocratie et de toutes ses valeurs analogues (droits de l'homme et diversité culturelle) extrêmement différente en 
Roumanie. Il en est ainsi car dans les esprits des intellectuels culturels, la démocratie demeure définie sur le plan ethnique, tandis que pour les intellectuels politiques, la voie vers un concept moderne et cosmopolite de la démocratie est déjà ouverte. Cette situation crée des ordres du jour différents à propos des questions importantes, telles que les droits des minorités nationales, de genre et sexuelles ainsi que la place de la Roumanie au sein de l'Europe institutionnalisée et les relations de la Roumanie avec la Hongrie et la République de Moldavie ${ }^{40}$.

\section{" Holocauste contre Goulag » : conservatisme et antisémitisme latent}

Quelques remarques préliminaires s'imposent. La disparition des juifs de la société roumaine n'a pas provoqué la disparition de l'antisémitisme ${ }^{41}$. L'objet de l'antisémitisme réside désormais dans la conspiration juive démoniaque invisible qui, selon les antisémites, contrôle des institutions telles que l'ONU, la CIA, le KGB et l'OTAN. Ainsi, la présence actuelle réelle des juifs n'est plus significative ni nécessaire dans les représentations antisémites. Les prédispositions du lecteur ou de l'auteur de textes antisémites à accepter ou reproduire des stéréotypes négatifs sont générés par la persistance de ces formes dans la tradition chrétienne orthodoxe, le folklore national, les souvenirs des pogromes passés, sous le dénominateur commun d'«antisémitisme latent $\aleph^{42}$. Toutefois, l'“antisémitisme militant" peut être trouvé dans le discours de la haine publié dans certains journaux: România Mare, édité par l'ultranationaliste Corneliu Vadim Tudor, Atac la persoană, édité par Dumitru Dragomir, Politica, Noua Dreaptă, Puncte Cardinale, Gazeta de Vest. En fait, de nombreux stéréotypes négatifs tels que "youpin", l'équivalent du roumain "jidan", pénètrent le discours politique courant. Néanmoins, de tels stéréotypes peuvent être interprétés comme une représentation de l'état général de l'anomie structurelle et des frustrations individuelles dans les circonstances du postcommunisme.

La relation entre le problème de l'antisémitisme latent et les intellectuels culturels et politiques intervient dans la polémique "Holocauste contre Goulag ", qui a divisé ces dernières années les deux générations d'intellectuels culturels et politiques, en dépit du remodelage répété des camps divergents et des modifications au sein de leurs rangs. Il faut également noter que ces discussions, sur un ton hautement passionné et qui se sont transformées parfois en attaques personnelles virulentes, tournent autour de prétendues "culpabilités" juives: l'accusation connue comme la "thèse judéobolchevique" selon laquelle les juifs sont responsables de l'instauration du communisme; en conséquence, les Juifs souhaitent cacher ce fait en cette période postcommuniste en mettant en avant la mémoire de l'Holocauste et en s'opposant ainsi à l'analyse et à la mémoire juste du communisme qui a pris la forme du Goulag avec ses victimes. Dans les deux cas, les Juifs sont collectivement, et par conséquent, ethniquement coupables, en premier lieu, pour le communisme (Goulag), puis pour prétendument nier le communisme par le biais de leur insistance sur l'Holocauste et ses liens épineux avec le régime de Ion Antonescu et ses légionnaires. Ainsi, une équation étrange est posée, une sorte de do ut des, où les Juifs sont perçus comme des négationnistes du communisme, tandis que de nombreux intellectuels culturels, consciemment ou non, se transforment en négationnistes de l'Holocauste.

27 Aujourd'hui, selon un observateur de la polémique en question digne de confiance, George Voicu, on peut affirmer qu'il existe un camp "pro-goulag" dont les protagonistes font une fausse comparaison entre les drames humains et historiques de 
l'Holocauste et du Goulag en les mettant sur le même plan, développant ainsi une position contradictoire établissant que l'entité collective juive est simultanément promotrice et négationniste du communisme. Selon Voicu, ce camp comprend Nicolae Manolescu, Gabriel Liiceanu, Dorin Tudoran, dissident audacieux sous Ceauşescu émigré aux États-Unis où il est devenu correspondant de la Voix de l'Amérique, Monica Lovinescu, fille de l'illustre Eugen Lovinescu émigrée en France où elle a courageusement dénoncé le régime de Ceauşescu au micro de Radio Free Europe, ainsi que divers journalistes, écrivains et critiques littéraires, tels que Alex ştefănescu, Constantin Ţoiu, tout deux regroupés autour de la revue România literară ${ }^{43}$, Ioan Buduca et de l'hebdomadaire 22, avec des voix hésitantes, Gabriela Adameșteanu et Gabriel Andreescu $^{44}$. On compte également des collaborateurs du Jurnal literar et d'Academia CaŢavencu. Parmi les personnes rejetant l'équivalence automatique entre l'Holocauste et le Goulag, ainsi que l'accusation selon laquelle le communisme aurait été mené par les juifs, et par conséquent le prétendu négationnisme juif du communisme, figurent Norman Manea, un survivant de l'Holocauste, écrivain émigré aux États-Unis, Zigu Ornea, historienne littéraire renommée, Vladimir Tismăneanu, professeur roumanoaméricain de sciences politiques à l'université du Maryland, Radu Ioanid, chercheur au musée de l'Holocauste à Washington D. C., Alexandra Laignel-Lavastine, politologue française, Michael Shafir, politologue et expert régional à Radio Free Europe, Stelian Tănase, Leon Volovici, critique et historien littéraire, chercheur au Centre d'étude de l'antisémitisme Vidal Sasson à l'université hébraïque de Jérusalem et Andrei Cornea, la plupart d'entre eux publiant principalement dans la revue Sfera Politicii et parfois dans l'hebdomadaire $22^{45}$. Il s'agit ici des protagonistes les plus représentatifs, leurs positions pouvant parfois fluctuer parce que les arguments politiques sont mêlés à des animosités personnelles et selon nous, à un possible manque de conscience du caractère antisémite des points de vue. En outre, il n'est pas question d'un débat académique puisqu'il est mené dans les journaux, les revues littéraires et polémistes, néanmoins son audience et ses conséquences politiques éventuelles peuvent être importantes étant donné le prestige prêté à ces intellectuels.

Dans le premier débat, « Holocauste contre Goulag » qui a donné son nom à l'ensemble de la polémique, les deux concepts divisent la presse démocratique roumaine en défenseurs et opposants de l'accusation de Roger Garaudy consécutivement à la publication en France de son ouvrage Les Mythes fondateurs de la politique israélienne ${ }^{46}$. L'auteur y soutient, dans un effort de minimiser la conscience de l'après-Shoah, que l'Holocauste a bénéficié d'une fructification exagérée. Sur la couverture de la traduction roumaine de son livre, on peut lire la devise : « ce n'est pas de ma faute [R. Garaudy] si ceux qui m'accusent sont particulièrement ceux qui ont organisé un commerce mondial de la vente des os de leurs grands-parents ». À l'arrière-plan du débat se situe Le livre noir du communisme $e^{47}$, publié en France en 1997, qui, en décrivant les formes de la dissidence anticommuniste dans le bloc soviétique et en comparant le communisme à l'Holocauste, a déclenché la discussion sur le thème du Goulag et sur l'équivalence entre Holocauste et Goulag. Dans ce débat, la victimisation et la compensation morale sont des thèmes politiques et rhétoriques primordiaux ${ }^{48}$. Il est alors étonnant de voir des intellectuels culturels importants, autoproclamés libéraux et démocrates, défendre les arguments de Garaudy niant l'Holocauste et accusant l'entité juive invisible de "confiscation de la mémoire » et de "monopole de la souffrance». Dans l'un de ses éditoriaux hebdomadaires, Nicolae Manolescu s'interroge : 
Une concurrence absurde est apparue parmi ceux qui, seulement aujourd'hui, dénoncent les horreurs du goulag d'une manière significative et ceux qui depuis des décennies dénoncent les horreurs de l'holocauste (...). C'est comme si la découverte $\mathrm{du}$ goulag allait remettre en question l'existence de l'holocauste. Est-ce que quelqu'un a peur de perdre le monopole sur la révélation des crimes contre l'humanité ? Le procès mené en France contre Garaudy, qui n'a jamais dit que l'holocauste n'avait jamais existé, mais qu'il y avait un lobby fantastique à son propos, est une raison indirecte soutenant ma supposition (...). Il est incorrect et immoral de faire taire des millions de victimes du communisme, par crainte que cela ne laisse pas suffisamment de personnes pour déplorer les victimes du nazisme ${ }^{49}$ (...). Je ne suis pas un antisémite si je souhaite débattre de l'holocauste et veut le situer correctement dans l'histoire du $\mathrm{XX}^{\text {ème }}$ siècle, en respectant la mémoire de ses victimes. Après tout, il n'est pas cynique de rechercher quel fut le nombre exact de victimes ${ }^{50}$. publication par les éditions Humanitas, fin 1996, du Journal de Mihail Sebastian, 1935-1941 (M. Sebastian étant le nom de plume de Iosif Hechter), dans lequel l'écrivain roumanojuif décrivit avec profondeur les moments les plus sombres de la Roumanie transfigurée par la Seconde Guerre mondiale, caractérisée par les caractères gothiques des légionnaires, les nazis et l'antisémitisme. Sa mort intervint brusquement en 1945 au carrefour entre le fascisme et le communisme. La découverte et la publication de ce journal en 1996, après 50 années de mystification communiste à propos de l'Holocauste juif, ont fait ressortir à la lumière du jour l'atmosphère chaotique de la guerre et les intimités fascistes de l'élite intellectuelle roumaine avec toute la saveur et le parfum de la gloire obscure passée. Sebastian décrit également dans son Journal la conversion aux valeurs du mouvement légionnaire de certains des intellectuels roumains les plus éminents du XXème siècle, ses amis de la Génération Critérium des années 1930. De nombreux intellectuels renommés entraînés dans le mouvement légionnaire revivent dans les pages du Journal, tels que Mircea Eliade, Emil Cioran et Constantin Noica, une génération dont nous avons déjà évoqué l'affiliation spirituelle et éthico-mystique à ce mouvement, plutôt que politique et criminelle. Avec sa sensibilité, le Journal révèle la triste et irréfutable évidence du Messianisme qui revendiquait à travers Nae Ionescu l'allégeance intellectuelle d'esprits sophistiqués au mouvement réactionnaire et à la politique du Rhinocéros ${ }^{51}$. À nouveau, cet événement a été l'occasion de la formulation de points de vue fâcheux de la part des intellectuels démocratiques, réitérant la symétrie entre Holocauste et Goulag, ou entre juif victime du nazisme et communiste ${ }^{52}$. C'est pourquoi, après avoir publié le Journal de Mihail Sebastian, la réflexion de Gabriel Liiceanu sur la foi des juifs après l'Holocauste dans les sociétés communistes, idée exprimée au cours d'un discours adressée à la communauté juive de Bucarest en 1997, demeure inconfortable dans la mesure où elle s'inscrit dans la thèse "judéobolchevique" : « comment est-il possible que celui qui a porté l'uniforme de la victime à un certain moment historique, endosse celui du bourreau? $\|^{53}$.

30

L'examen détaillé de tels points de vue par Norman Manea établit que «l'analogie ne laisse aucun espace pour une réelle condamnation de l'antisémitisme et de l'Holocauste, ou pour une analyse honnête de la "culpabilité heureuse" de certains intellectuels roumains brillants comme Eliade, Cioran, Nae Ionescu, Noica ». Répondant également à une série d'articles écrits par Nicolae Manolescu, Manea attire notre attention sur l'incompatibilité entre les valeurs démocratiques et libérales de Manolescu et sa propension à des sympathies littéraires fascistes : «dans un éditorial 
portant le titre - la chasse aux sorcières - le directeur de la revue importante România literară décrie l'aversion public prolongée contre Céline et Hamsun [philo-nazis et antisémites], la campagne "israélienne" contre Eliade, le débat récent en France concernant le passé légionnaire de Cioran, ainsi que les exagérations contenues dans le Journal de Sebastian ${ }^{54}$. Il y a aussi d'autres débats appartenant à la polémique « Holocauste contre Goulag ». L'un d'entre eux implique le poète national, Mihail Eminescu ${ }^{55}$. Dans ce cas, la polémique réside dans la critique culturel du travail du poète romantique qui jusqu'à récemment n'a jamais été approché dans un esprit critique en raison de sa mythification post mortem, de son appartenance à la lignée nationaliste et de ses écrits journalistiques antisémites occasionnels ${ }^{56}$. D'autres débats connexes tournent autour de la même problématique, comme la polémique à propos de la publication dans le quotidien français Le Monde (du 28 janvier 1998) de la tribune de Radu Ioanid et d'Alexandra Laignel-Lavastine réprouvant les tentatives répandues de réhabilitation du maréchal Antonescu à travers la Roumanie ${ }^{57}$. D'autres discussions mettent l'accent sur comment faire la distinction parmi les partisans qui combattirent le communisme dans les montagnes, alors que dans leurs rangs on comptait des légionnaires (par exemple, le cas du «Mémorial Sighet $»^{58}$ et le "rassemblement du Musée du paysan roumain ${ }^{59}$, consacrés aux partisans) ${ }^{60}$. Avec la traduction et la présentation dans la presse de l'article de George Voicu « Reacţia de prestigiu » (« La réaction de prestige ») fin 1999début 2000 , le débat s'est déplacé en direction des publications françaises renommées, Le Monde ${ }^{61}$ et Les Temps Modernes ${ }^{62}$. On en trouve un écho dans les numéros les plus récents de l'hebdomadaire roumain 22 , sur un ton très émotionnel, avec des incompréhensions, des vengeances personnelles et des positionnements confus ${ }^{63}$.

L'analyse des débats "Holocauste contre Goulag» montre que, certains des intellectuels culturels de l'ensemble GDS / Humanitas / disciples de Noica, font preuve de peu de connaissances historiques et de compréhension pour l'altérité, acceptant des formulations essentialistes sur la judéité et la conspiration intellectuelle, le judéobolchevisme, la confiscation de la mémoire et le négationnisme du communisme / goulag ${ }^{64}$, tandis qu'ils soutiennent une fausse comparaison en admettant la symétrie automatique entre les deux drames historiques : l'Holocauste et le Goulag. Analysant la signification de ces débats, George Voicu révèle :

Les carences de culture démocratique de bon nombre de ceux qui font l'opinion publique dans notre pays, par ailleurs des spécialistes reconnus dans leurs domaines, ont finalement eu le dessus, et le témoignage de Sebastian a été peu à peu minimisé, réduit à un point de vue quelconque, d'une valeur dans le meilleur des cas limitée.(...) La polémique s'est beaucoup développée et de façon assez chaotique, mais on peut néanmoins distinguer la récurrence de certains sujets: l'engagement d'une série d'intellectuels parmi les plus brillants du côté de l'extrême droite dans la période de l'entre-deux-guerres et, en réponse, la collaboration d'autres, plus nombreux, avec le régime communiste ; l'évocation de la Shoah et, par souci de symétrie, l'évocation du Goulag. À partir de là, la polémique s'est étendue à des sujets propres à notre actualité politique, mais visiblement liés aux précédents : les poussées racistes, xénophobes et antisémites dans la société roumaine d'aujourd'hui; le problème de la réhabilitation du maréchal Antonescu (et de certains ministres de son gouvernement); notre position face à l'Occident, etc.(...) Après la Shoah, l'antisémitisme est perçu par la quasi-totalité des intellectuels roumains comme une idéologie compromettante, qui doit être blâmée (même les antisémites notoires refusent de se considérer comme tels, bien que leurs subterfuges n'échappent à personne). Mais des attitudes, des 
idées et des mécanismes intellectuels à potentiel antisémite sont perceptibles encore aujourd'hui, en fin de millénaire, même parmi nos élites culturelles. La polémique autour de la Shoah, dont les intellectuels roumains, dans leur écrasante majorité, ne reconnaissent pas la spécificité, en est la preuve. (...) Invoquer Roger Garaudy comme une autorité en la matière, quitte à falsifier ses thèses pour les rendre plus "présentables", dénote un manque de discernement inquiétant sur le plan des idées et sur celui des valeurs. Une culture démocratique, du point de vue intellectuel, mais aussi sociologique, ne peut se construire sur de telles bases.(...) De là à considérer la Shoah comme un stratagème permettant aux Juifs de s'assurer «le monopole profitable de la souffrance »- comme le clament Radu Theodoru et Corneliu Vadim Tudor, leader du parti « La Grande Roumanie », la distance est-elle énorme? L'équivalence arbitrairement établie entre Shoah et Goulag est généralement renforcée par une autre, plus large, entre fascisme et communisme. (...) Or, l'antisémitisme des intellectuels roumains est un problème de la culture roumaine, et un problème non pas secondaire, mais essentiel. ${ }^{65}$

À la lumière de leurs valeurs, il apparaît clairement qu'il est impossible de qualifier les intellectuels culturels simplement de nationalistes, de la même façon que les ultranationalistes le sont considérés. De fait, il serait trop simpliste de considérer aujourd'hui certains intellectuels culturels comme des antisémites purs. Cela est vrai dans la mesure où les centres d'intérêt de ces intellectuels sont complètement différents de ceux des ultranationalistes dont l'antisémitisme est explicite et militant. En ce sens, leur attitude politique et culturelle est chargée d'une forme d'antisémitisme subtile et parfois confuse, fluctuant entre un antisémitisme « latent ", « utilitariste " voire non intentionnel. Toutefois, cela n'est pas sans conséquences politiques significatives dans un pays où l'ouvrage Le protocole des sages de Sion est diffusé et vendu librement dans les rues ${ }^{67}$. Le fait que certains intellectuels aient donné libre cours à ce genre de rhétorique maladroite peut être expliqué par plusieurs raisons. L'une d'entre elles est le manque d'introspection critique à l'égard du communisme expliqué par la coalition nationaliste-communiste ou "rouge-brun", nostalgique du régime précédent, au pouvoir jusqu'en 1996. Au contraire, les efforts d'intégration à l'Europe et à l'OTAN entre 1996 et 2000 ont déplacé l'objet de l'attention en matière politique du passé vers l'avenir. Une autre des raisons principales, selon Matei Călinescu ${ }^{68}$, réside dans le souhait authentique de ces intellectuels culturels de trouver une solution à l'identité fragmentée de la culture roumaine. Depuis Cioran, cette identité est perçue par les intellectuels roumains, vivant dans un complexe d'infériorité manichéen indigne, comme "mineure" en comparaison aux cultures "majeures" d'Europe occidentale. Par ailleurs, les débats montrent également que le concept d'Holocauste, en tant que mémoire organisée, et le fascisme ont été minimisés sous l'idéologie communiste ${ }^{69}$ et qu'ils n'ont pas fait l'objet d'un examen critique après $1989^{70}$. Dans de telles circonstances, les thèses négationnistes concernant l'Holocauste juif ou le prétendu négationnisme du communisme deviennent problématiques dans la mesure où ils flottent dans le contexte général $d u$ renouveau de l'antisémitisme et du fondamentalisme orthodoxe accru à travers les sociétés d'Europe de l'est en transition.

À la lumière de ces débats, le concept d'intellectuel démocratique roumain souffre d'un déplacement de sens. 


\section{Conclusion : réflexions sur la démocratie des intellectuels roumains aujourd'hui}

Des nuances symboliques dans les valeurs centrales des intellectuels culturels devaient être notées dans la mesure où elles constituent le fondement du pouvoir entre 1996 et 2000. En 1989, un intellectuel démocratique était plus particulièrement un anticommuniste, une définition explicable par le changement brusque de régime politique. Cette définition fut complétée par l'opposition avec l'émergence des ultranationalistes $^{71}$. L'intellectuel démocratique était également intéressé par la question des droits de l'homme élémentaires reniés précédemment (libertés de voyager, d'association, de conscience, de la parole, droit à l'avortement, suppression de la peine de mort). En outre, le sens de la démocratie s'est élargi en incluant des valeurs politiques occidentales, à savoir orientées vers le libéralisme économique et politique ainsi que la société civile. Entre 1990 et 1996, ces valeurs constituaient le capital de l'opposition au gouvernement, la seconde génération des intellectuels culturels. L'identité nationale roumaine était communément conçue dans une orientation ou une ré-identification à l'Europe, mais pour les intellectuels culturels il s'agissait plus d'un rapprochement de l'héritage culturel européen plutôt que de sa tradition politique, situation explicable par leur formation à dominante philosophique et littéraire.

Néanmoins, lorsque les questions des minorités nationales et de la diversité culturelle sont devenues tendues, ces intellectuels démocratiques, quoique culturellement proeuropéens, ont échoué dans la reconnaissance du fait que leur modèle théorique était dépassé et tributaire du concept national traditionnel de l'identité, toujours défini en termes ethniques et religieux. Des revendications émanaient des minorités hongroise, rom et juive, mais également de groupes d'orientation sexuelle. Lorsqu'ils en eurent le plus besoin, (au moment de leur arrivée au pouvoir en 1996), la culture ne put fournir aux intellectuels culturels des arguments modernes, séculiers, démocratiques et libéraux pour gouverner. Au contraire, la culture roumaine, en tant que source de légitimité de leur politique, a renforcé ses dimensions conservatrice, religieuse, élitiste, pseudo-démocratique, monarchiste, voire antirépublicaine. À la lumière de ces caractéristiques et de ces débats, il semble qu'au lieu de constater le caractère démocratique et libéral autoproclamé de tant d'intellectuels culturels roumains, nous observons le conservatisme à l'œuvre au sein de paradigmes démocratiques modernes dont la signification est dévaluée. En l'absence de tradition républicaine, démocratique et libérale roumaine, la préférence politique des intellectuels culturels penche largement vers une monarchie constitutionnelle dont ils aimeraient certainement demeurer le corps consultatif.

Cependant, ce conservatisme ne dépasse pas la politique du XIX ${ }^{\text {ème }}$ siècle dépourvue de contenus démocratiques, lorsque ses protagonistes revendiquent, par exemple, la suppression du suffrage universel et égalitaire pour les Roumains ou débattent de façon péjorative des minorités tenues en dehors de la citoyenneté définie en termes exclusifs. Par ailleurs, les valeurs des intellectuels culturels correspondent à un nationalisme modéré, à un désir d'intégration globale et européenne, parfois mêlé de nostalgie pour une forme d'autochtonie, telle que définie par Noica. Au milieu d'eux, on trouve les intellectuels politiques, dont les valeurs sont simultanément interconnectées et différentes, tentant de diffuser le concept de démocratie dans une approche libérale et dans un effort de mettre l'accent sur la démocratie reposant sur une définition 
cosmopolite et civique et non sur l'identité nationale. Toutefois, cette nouvelle forme de démocratie n'a pas été absorbée par l'opinion publique en raison, principalement, du climat politique et culturel conservateur, de la résurgence des mythes nationalistes et des conceptions de la roumanéité de l'entre-deux-guerres, ainsi que de l'absence en Roumanie de tradition de la modernité séculaire. De surcroit, la prédominance des modèles culturels du XIXème siècle dans la vie politique roumaine actuelle lui confère un statut, "sur le plan stylistique éminemment baroque ", comme le suggère Adrian Marino, « sur un fond d'improvisation historique continue $»^{72}$.

\section{NOTES}

1. Le concept d'antisémitisme repose ici sur la politique du Centre d'études de l'antisémitisme Vidal Sassoon de l'université hébraïque de Jérusalem: dans la mesure où il n'existe pas de "sémitisme" en tant qu'idéologie, il n'y a pas d'“antisémitisme" en tant que contre-idéologie, mais il existe uniquement l'antisémitisme, en tant qu'idéologie qui depuis le début se réfère à l'antagonisme racial et ethno-religieux à l'égard des Juifs.

2. Verdery (Katherine), National Ideology Under Socialism. Identity and Cultural Politics in Ceauşescu's Romania, Berkeley: University of California Press, 1991 ; Verdery (Katherine), What Was Socialism, and What Comes Next ?, Princeton : Princeton University Press, 1996.

3. Zeletin (ştefan), Burghezia română, Bucarest: Editura Cultura NaŢională, 1925 ; Zeletin (ştefan), Neoliberalismul. Studii asupra istoriei şi politicei burgheziei române, Bucarest: Editura "Pagini agrare şi sociale", 1926 ; Lovinescu (Eugen), Istoria civilizaŢiei române moderne (vols. I, II, III), Bucarest : Editura Ancora, 1924-1926.

4. Weber (Max), The Theory of Social and Economic Organization (ed. Talcott Parsons), New York: The Free Press, 1947.

5. Voir également Volovici (Leon), National Ideology and Antisemitism. The Case of the Romanian Intellectuals in the 1930s, Oxford : Pergamon Press, 1991, p. 71.

6. Pour un point de vue critique soulignant la corruption et le clientélisme de l'élite politique roumaine entre 1996 et 2000, voir Stan (Lavinia), «Do Ut Des: Political Clientelism and Corruption in the Balkans » (mimeo).

7. Crainic (Nichifor), Ortodoxie şi etnocraţie, Bucarest, 1937.

8. Bulei (Ion), «Conservatorismul românesc », in Mungiu-Pippidi (Alina), ed., Doctrine politice. Concepte universale şi realităţi româneşti, Iaşi : Polirom, 1998, pp. 84-93.

9. Voir aussi Mungiu-Pippidi (Alina), «Romanian Political Intellectuals before and after the Revolution », in Bozóki (András), ed., Intellectuals and Politics in Central Europe, Budapest : CEU Press, 1999, p. 93.

10. Garton Ash (Timothy), « Does Central Europe Exist?», in Schöpflin (G.), Wood (N.), eds., In Search of Central Europe, Totowa : Barnes and Noble, 1989.

11. Wandycz (Piotr), The Price of Freedom: A History of East-Central Europe from the Middle Ages to the Present, London : Routledge, 1992 and Āgh (Attila), The Politics of Central Europe, Sage Publications, 1998.

12. Kundera (Milan), "The Tragedy of Central Europe », New York Review of Books, 26/04/94. Cependant, le divorce déterministe culturel et subtilement dérogatoire entre l'Europe du centreest et les Balkans et la Russie, tel que Wandycz et Kundera l'entendent, ne peut échapper à notre 
attention. À propos d'une opinion similaire, voir Todorova (Maria), Imagining the Balkans, Oxford / New York : Oxford University Press, 1997.

13. Boia (Lucian), Istorie şi mit în conştiinţa românească, Bucarest: Humanitas, 1997 ; Wolff (Larry), Inventing Eastern Europe. The Map of Civilization on the Mind of the Enlightenment, Stanford : Stanford University Press, 1994 ; Bideleux (Robert), Jeffries (Ian), A History of Eastern Europe. Crisis and Change, London : Routledge, 1998 ; Hyde-Price (Adrian), The International Politics of East Central Europe, Manchester: Manchester University Press, 1996; Holmes (Leslie), PostCommunism. An Introduction, Durham : Duke University Press, 1997.

14. Voir également le texte de Durandin (Catherine), in Răileanu (Petre), Fin de siècle, un nou început, Bucarest : Editura Atlas, 1999, pp. 32-42.

15. Pour une critique de la théorie occidentale sur les intellectuels dissidents et son échec empirique sous le postcommunisme et dans les Balkans, voir, Stan (Lavinia), «The Unbearable Lightness of Being an Eastern European Intellectual », Government and Opposition, 35 (2), Spring 2000.

16. Marino (Adrian), Politică şi cultură. Pentru o nouă cultură română, Iaşi : Polirom, 1996, p. 153.

17. Cette étude n'aborde que certains aspects relatifs aux disciples de Noica dans le climat politique et culturel actuel en Roumanie. Il ne s'agit donc pas d'une approche critique du travail précieux du philosophe Constantin Noica et de ses disciples érudits, G. Liiceanu and A. Pleşu. On peut également noter une distinction entre les intellectuels culturels et les disciples de Noica, dans le sens où ces derniers constituent un groupe au sein du premier.

18. À propos des écrits et du passé légionnaires de Noica, voir Ioanid (Radu), The Sword of the Archangel. Fascist Ideology in Romania, New York : East European Monographs, 1990, pp. 144-145. À propos de l'affiliation spirituelle, éthico-mystique, plutôt que politique ou criminelle au mouvement légionnaire concernant Noica et Eliade, voir Volovici (Leon), op.cit., pp. 134-137.

19. Tismaneanu (Vladimir), Pavel (Dan), « Romania's Mystical Revolutionaries : The Generation of Angst and Adventure Revisited », East European Politics and Societies, 8 (3), 1994.

20. Physicien de formation, H.-R. Patapievici est devenu connu avec son ouvrage Politice (Patapievici (Horia-Roman), Politice, Humanitas, 1996) bien qu'il ait précédemment publié une nouvelle sensible décrivant ses expériences intellectuelles, Zbor în bătaia săgeŢii, récompensé par le prix de l'Union des écrivains. Il est également membre du GDS et éditeur aux éditions Humanitas.

21. Patapievici (Horia-Roman), op.cit., pp. 62-68. Voir la critique politique incisive des idées de Patapievici dans : Miroiu (Mihaela), «Misera plebs "Societatea retro, Bucarest : Editura Trei, 1999, pp. 113-133.

22. Patapievici (Horia-Roman), « Comunismul american », in Patapievici (Horia-Roman), op.cit., pp. 131-132.

23. Patapievici (Horia-Roman), «Deriva ideologică », 22, (21), 1998, qui a reçu des réponses qualifiées de Andreescu (Gabriel), «Despre drepturile omului, ecologie, feminism. Pe marginea unui articol de Horia Patapievici », 22, (23), 1998 et de Miroiu (Mihaela), "Antifeminism şi Conservatorism", 22, (40), 1998.

24. Patapievici (Horia-Roman), « Problema sindicală », 22, (14), 1999.

25. Patapievici (Horia-Roman), « Minoritatile imperiale», 22, (38), 1998. Voir les réactions à ce texte dans Andreescu (Gabriel), Molnár (Gusztàv), Problema transilvană, Iaşi : Polirom, 1999.

26. Antonesei (Liviu), « În jurul “cazului” Patapievici », Sfera politicii, (40), 1996.

27. Manolescu (Nicolae), « Votul universal », România literară, (23), 1996.

28. Telles étaient les invectives de Pruteanu (George), «Eşecurile împotrivirii », Dilema, (173-174), 1996 ou de Popescu (C. T.), «Cum să ne descotorosim de poporul român », Adevărul, (1869), 17/06/96.

29. Tismăneanu (Vladimir), Fantasies of Salvation. Democracy, Nationalism, and Myth in PostCommunist Europe, Princeton : Princeton University Press, 1998, note 29, p. 176. 
30. Haddock (Bruce), Caraiani (Ovidiu), « Nationalism and Civil Society in Romania », Political Studies, 47, 1999.

31. L'approche de la maison d'édition Humanitas doit être comprise à la lumière de la question soulevée ici, à savoir celle des "disciples de Noica" et de la publication non-critique de certains philosophes mystiques roumains et allemands. La contribution précieuse de cette maison d'édition au climat culturel roumain dans son ensemble ne doit pas être sous-estimée.

32. En Occident, des approches critiques de Nietzsche et de Heidegger ont été écrites il y a deux décennies dans plusieurs publications : les traductions de Nietzsche de Walter Kaufmann ou les traductions d'Heidegger de William Lovitt; voir aussi Leo Strauss (Leo), Cropsey (Joseph), History of Political Philosophy, Chicago : The University of Chicago Press, 1987 et Guignon (Charles B.), ed., Cambridge Companion to Heidegger, Cambridge : Cambridge University Press, 1993, pour ne mentionner que quelques ouvrages.

33. À propos des regrets de Cioran, voir Volovici (Leon), op.cit. et Călinescu (Matei), « Ionesco and Rhinoceros: Personal and Political Backgrounds », East European Politics and Societies, 9 (3), pp. 393-432.

34. Pour une analyse nuancée de l'approche d'Eliade à l'égard du mouvement légionnaire, doutant justement du prétendu caractère de "droite" de la conception d'Eliade des mythes, voir Volovici (Leon), op.cit., pp. 145-146.

35. Andreescu (Gabriel), ed., NaŢionalişti, antinaŢionalişti. O polemică în publicistica românească,Iaşi : Polirom, 1996.

36. L'exclusion au début de l'année 2000 de Gabriel Andreescu et Dan Pavel du GDS et de son hebdomadaire 22 est un signe éloquent que le processus de désagrégation au sein du GDS se développe sur la base des lignes mentionnées dans cet article: les conservateurs contre les progressistes.

37. Voir également Stan (Lavinia), « Romanian Political Science Since 1989 », European Journal of Political Science, (35), 1999.

38. Haddock (Bruce), Caraiani (Ovidiu), art.cit.

39. Metro Media Transilvania, « Barometrul de opinie publică », Roumanie, mai 1999.

40. Voir Andreescu (Gabriel), NaŢionalişti, antinaţionalişti (op.cit.).

41. Selon le dernier recensement de 1992, aujourd'hui il y a moins de 9107 juifs, soit 0,3\% de la population totale. Moroianu-Zlătescu (Irina), Oancea (Ioan), The Legislative and Institutional Framework for National Minorities from Romania, Bucarest: The Romanian Government/ The Council for Ethnic Minorities, 1994.

42. Volovici (Leon), «Antisemitism in Post-Communist Eastern Europe: A Marginal or Central Issue ?», ACTA, 5, 1994, p. 3 ; Volovici (Leon), « Mit şi realitate», Sfera Politicii, 32, 1995, p. 4 ; Volovici (Leon), «Notes on Latent Antisemitism», The Vidal Sassoon International Center for the Study of Antisemitism. Annual Report, 1997, pp. 16-18.

43. À propos des points de vue de Nicolae Manolescu, de Dorin Tudoran et de România literară en général, voir le numéro de 1999 de Jewish Policy Research de Grande Bretagne, (http://www.jpr.org.uk/antisem/countries/romania/romania.htm).

44. Andreescu (Gabriel), « Roşu, negru », 22, (37), (38), (41), (42) et (43), 1998.

45. L'essence de ce débat peut être trouvée dans un article signé par G. Voicu (Voicu (George), « Reacţia de prestigiu. Reflecţii pe marginea unei polemici », Sfera politicii, (63), 1998).

46. Publié en Roumanie par la maison d'édition ALMA TIP en 1998. La postface signée par George Dănescu est également antisémite.

47. Traduit en roumain et publié par Humanitas en 1998.

48. À propos de ce débat toujours en cours, se référer à la presse : Manolescu (Nicolae), «Holocaust şi Gulag », România Literară, (9), 1998 ; « Roşu şi negru », România literară, (23-24), 1998 ; Adameşteanu (Gabriela), Palade (Rodica), « Fascism şi comunism », 22, (7), 1998 ; Cornea 
(Andrei), « Accident sau simptom », 22, (25), 1998 ; Ornea (Zigu), « Polemici şi revizuiri », 22, (17), 1998 ; Shafir (Michael), «O tragicomedie în desfăşurare? », Sfera Politicii, (61), 1998 ; Neumann (Victor), « Despre Holocaust şi Gulag », Sfera Politicii, (61), 1998.

49. Manolescu (Nicolae), « Holocaust şi Gulag » (art.cit.).

50. Manolescu (Nicolae), « Ce inseamnă să fii rasist », România literară, (19), 1998.

51. Rhinocéros fait référence à la pièce de 1958 d'Eugène Ionesco portant le même titre. L'auteur franco-roumain, d'origine juive, comme le révèle le journal de Mihail Sebastian, y fait le récit indirect et métaphorique de ses expériences roumaines dramatiques liées à la montée du mouvement légionnaire et relatant la façon dont des intellectuels roumains célèbres y ont succombé dans un processus de "rhinoceritis".

52. À propos de ce débat en cours, se référer à la presse: Shafir (Michael), art.cit.; Ornea (Zigu), « Opinitiri împotriva Jurnalului lui Sebastian », Dilema, (226), 1997 ; Ornea (Zigu), « Unde duc înverşunarea şi excesul de zel », Dilema, (288), 1998 ; Călinescu (Matei), «"How can one be what one is?": Cioran and Romania ", in Zub (Al.), ed., Identitate si Alteritate, Iaşi : Editura UniversităŢii “Alexandru Ioan Cuza", 1996 ; Liiceanu (Gabriel), « Sebastien, mon frère », 22, (19), 1997 ; Manea (Norman), «IncompatibilităŢile », 22, (23), 1998 [publié initialement dans The New Republic, 20/04/98]; Bell (Daniel), "A Rediscovered Writer Opens Wounds of the Past", Newsletter. Committee on Intellectual Correspondence, (3), winter 1998/1999.

53. Liiceanu (Gabriel), « Sebastien, mon frère » (art.cit.).

54. Manea (Norman), « IncompatibilităŢile » (art.cit.).

55. À propos de ce débat, se référer à la presse: TiŢa Chiper dialoguant avec A. Paleologu (Paleologu (Alexandru), «Imaginile succesive », Dilema, (265), 1998); Rădulescu (Răzvan), «Eminescu văzut de departe », Dilema, (265), 1998 ; Bobe (T. O.), «Poezie, haine grele », Dilema, (265), 1998; Ornea (Zigu), « Poetul naŢional», Dilema, (265), 1998 ; Teodorescu (Cristian), "Judecarea lui Eminescu », România literară, (11), 1998 ; Munteanu (George), " Cine, de ce şi cum se desparte de Eminescu », Adevărul literar şi artistic, 16 iunie, 1998.

56. À propos du racisme d'Eminescu et de son antisémitisme économique, plutôt qu'authentique, voir Volovici (Leon), op.cit. et Ioanid (Radu), op.cit.

57. À propos de la chronologie des initiatives pour élever des bustes d'Antonescu dans diverses villes de Roumanie (Slobozia, Tîrgu-Mureş, Piatra NeamŢ ou le cas duplice de la garnison militaire de Lugoj) depuis le début des années 1990, ainsi que des débats dans la presse et des résolutions gouvernementales et judiciaires, voir Voicu (George), «Rechizitoriu cu tîlc ", Sfera Politicii, (32), 1995 ; Volovici (Leon), art.cit. ; Braham (Randolph L.), Romanian Nationalists and the Holocaust: The Political Exploitation of Unfounded Rescue Accounts, New York: The Rosenthal Institute for Holocaust Studies / City university of New York, 1998, pp. 61-70.

58. Musée du Goulag roumain, en partie financé par le Conseil de l'Europe (NdT).

59. Début décembre 1997, le Musée du paysan roumain a consacré une journée d'hommage à Corneliu Zelea Codreanu, l'ancien chef de la Garde de fer. Ce rassemblement était supposé commémorer la résistance anticommuniste des années 1950 (NdT).

60. Shafir (Michael), art.cit. ; Adameşteanu (Gabriela), Palade (Rodica), art.cit.

61. Reichmann (Edgar), «Contre les purificateurs de l'Histoire en Hongrie et en Roumanie », Le Monde, 15/01/00 ; Liiceanu (Gabriel), « L'Antisémitisme en Roumanie », Le Monde, 15/02/00.

62. Voir l'article de Braham (Randolph L.), «Offensive contre l'Histoire: les nationalistes hongrois et la Shoah ", Les Temps Modernes, (606), novembre-décembre 1999,et la traduction en français du texte de Voicu (George), «Reacţia de prestigiu », dans Les Temps Modernes, (606), novembre-décembre 1999.

63. Voir les textes « Un text din Le Monde si replici la el, I-VII », 22, (5), (6), (7), (11), (12), (14), et (16), 2000. Pour une meilleure compréhension de ce changement, voir Voicu (George), « Indecenţa comparativă », 22, (11), 2000. 
64. À propos de ce débat, voir également Shafir (Michael), Radical Politics in Post-Communist Central and Eastern Europe (à paraître), cité avec la permission de l'auteur ; Shafir (Michael), «The Man They Love to Hate: Norman Manea Between Holocaust and Gulag ", East European Jewish Affairs (à paraître) ; Cornea (Andrei), « Holocaustul la tribunal », 22, (21), 2000.

65. Voicu (George), « Reacţia de prestigiu » (art.cit.). Nous reprenons ici la traduction française de ce texte (par Sandra Dumitrescu) parue dans Les Temps Modernes. Le texte a été publié sous le titre : « L'honneur national roumain en question » $(\mathrm{NdT})$.

66. Shafir (Michael), «Radical Politics in East-Central Europe. Part III : X-Raying PostCommunist "Radical Minds". C) Conspiracy Theories and Anti-Semitism », RFE/RL East European Perspectives, 2 (1), 2000.

67. La traduction de l'ouvrage LeProtocole des sages de Sion est signée de Ioan Moţa et a été publiée par la maison d'édition Samizdat en 1999. Ce livre a été publié auparavant par d'autres maisons d'édition, la première étant Oblio, d'Oradea.

68. Călinescu (Matei), «How Can One be ?» (art.cit.) ; Călinescu (Matei), «Despre culturile mici », Dilema, (281), 1998 ; Călinescu (Matei), « Ionesco et Rhinoceros : Personal and Political Backgrounds » (art.cit.).

69. Florian (Alexandru), "The Holocaust in Romanian Textbooks ", in Braham (Randolph L.), ed., The Tragedy of Romanian Jewry, New York: The Rosenthal Institute for Holocaust Studies-The City University of New York, 1994 ; Volovici (Leon), « Antisemitism in Post-Communist Eastern Europe » (art.cit.), p. 13.

70. Toutefois, des changements significatifs sont à relever dans les nouveaux manuels d'histoire (publiés par les éditions Humanitas, Nemira et Corint) consacrant des textes et des illustrations à l'Holocauste et reprenant même des citations du Journal de Sebastian.

71. En fait, anticommunisme est un terme générique couvrant les socio-démocrates, les libéraux, les conservateurs et les fascistes. En 1989, et immédiatement après, l'anticommunisme constituait un thème suprême occultant ces ombres idéologiques intérieurs, ayant toutes réémergé dans la Roumanie actuelle.

72. Marino (Adrian), op.cit., p. 77. 